\title{
Mathematical Modelling to Simulate Biological Fluid Flow in a Collapsible Tube
}

\author{
Muhammad Zeeshan Ashraf ${ }^{1}$, Muhamad Riaz Khan ${ }^{2}$, Shahzad Waheed ${ }^{3}$, Muhammad Ahsan ${ }^{4}$, \\ Saira Hussnain ${ }^{4}$
}

${ }^{1}$ Department of Basic Sciences \& Humanities, University of Engineering and Technology, Lahore, Pakistan

${ }^{2}$ Department of Mathematics, Lahore Garrison University, Lahore, Pakistan

${ }^{3}$ Department of Mathematics, University Of Lahore, Lahore, Pakistan

${ }^{4}$ Department of Mathematics, Hajvery University, Lahore, Pakistan

\section{Email address:}

m.zeeshan@uet.edu.pk (M. Z. Ashraf), riaz.younas@lgu.edu.pk (M. R. Khan), rswaheed@yahoo.com (S. Waheed), ahsan_maths@yahoo.com (M. Ahsan), sairahusnain@ymail.com (S. Hussnain)

\section{To cite this article:}

Muhammad Zeeshan Ashraf, Muhamad Riaz Khan, Shahzad Waheed, Muhammad Ahsan, Saira Hussnain. Mathematical Modeling to Simulate Biological Fluid Flow in a Collapsible Tube. American Journal of Mechanics and Applications. Vol. 6, No. 1, 2018 , pp. 1-6. doi: 10.11648/j.ajma.20180601.11

Received: December 26, 2017; Accepted: January 11, 2018; Published: January 29, 2018

\begin{abstract}
The purpose of this paper is to advance a mathematical model for reviewing to simulate biological flows such as blood flow in arteries or veins, flow of urine in urethras and air flow in the bronchial airways. They can also be used to study and prediction of many diseases, as the lung disease (asthma and emphysema), or the cardiovascular diseases (heart stroke), Makinde (2005). In this work, laminar flow of an incompressible viscous fluid through a collapsible tube of circular cross section is considered. Collapsible tubes are easily deformed by negative transmural pressure owing to marked reduction of rigidity. Thus, they show a considerable nonlinearity and reveal various complicated phenomena Our objectives are to study the effect of temperature along the tube as the fluid Prandtl number and Reynolds number increases. We launch the mathematical formulation of the problem. The problem is solved by using power series and perturbation techniques with help of boundary conditions and results are displayed graphically for different flow characteristics, velocity profile.
\end{abstract}

Keywords: Power Series, Transmural Pressure, Collapsible Tube

\section{Introduction}

In human body, all the channels are malleable and also collapsible. That is, when the exterior pressure exceeds the interior pressure, the tube cross-sectional area can be deliberately shortened, if not fully reduced. In this paper, a mathematical model to simulate biological fluid flow in a collapsible tube is presented. Methodical solutions are made for the problem using perturbation technique. The computer extension of the resulting power series solutions, its analysis and analytic extension, We obtained accurately a turning point $\mathrm{Rc}$ ( $\mathrm{R}$ the flow Reynolds number), as well as the asymptotic performance of the skin friction and fluid pressure gradient as $\mathrm{R} \rightarrow 0$ on the secondary solution branch. The model is most appropriate to simulate wind tunnel tests on rheological phenomenon in physiological systems. is a narrowing of any tubular structure in the body, including blood vessels, heart valves, vertebral canal and the GI area. Blood vessel narrowing is one of the more common usages of this term. High cholesterol could contribute to the stenosis of an artery when it accumulates on the inner wall of the artery. This accretion, referred to as atherosclerosis, can build up within the artery to the point where it reduces blood flow to organs of the body. When blood flow is reduced, nutrients and oxygen cannot travel to the tissues that need it. Below are a series of illustrations that will help us to understand the process of atherosclerosis (vascular disease) and the kinds of problems that can arise in this condition:

The major research goal remains, the full understanding of the flow structure and the mechanism driving this flow. Many previous theoretical works on flow in collapsible tubes concentrated on the development and analysis of simpler 
models, by reducing the spatial dimension of the problem, which involve a number of ad-hoc assumptions e.g., Contrad (1969), Grotberg (1971), Flaherty et al. (1972), Cowley (1982), Bonis \& Ribreau (1987), etc. Experimental example of the work that have been done on collapsible tube includes the one performed with finite-length elastic tubes whose upstream and downstream ends are held open (i.e., Starlingresistor, Brower \& Scholten 1975, Bertram 1986). Inside a pressure chamber, thin-walled elastic tube (made of latex rubber) is mounted on two rigid tubes. Fluid (liquid or gas) typically water or air respectively is driven through the tube, either by applying a controlled pressure-drop between the ends of the rigid tubes or by controlling the flow rate. If the external presssure exceeds the fluid pressure by a sufficiently large amount, the tube buckles non-axisymmetrically, which then leads to a nonlinear relation between pressure-drop and flow rate. At sufficiently large Reynolds numbers, the system produces self-excited oscillations, and exhibits hysteresis in transitions between dynamical states, multiple modes of oscillations (each having distinct frequency range), rich and complex nonlinear dynamics (Bertram et al. 1990). The inertia and resistance of the fluid in the supporting rigid tubes have an important influence on the system's overall dynamics. This experiment forms the basis for most recent theoretical investigations due to its three-dimensional nature. Meanwhile, Bertram and Pedley (1982), Bertram and Raymond (1991) investigated two-dimensional channel theoretical model with one wall of the channel been replaced by a membrane under longitudinal tension, viscous flow is driven through the channel by an imposed pressure-drop. The variation between the external pressure and the internal flow determine the deformation of the membrane. The dynamics of the problem is described by nonlinear ODE's whose numerical solutions exhibit oscillatory behaviour reminiscent of that observed in experiments. Despite the difficulties of producing two-dimensional flows experimentally, this system still attracted considerable theoretical attentions. Since it avoid the complications of three-dimensional flows found in the Starling-resistor, while still exhibiting phenomena such as flow limitation and self-excited oscillations.

Meanwhile, mathematical model of physical phenomena often results in nonlinear equations for some unknown function. Usually the problems cannot be solved exactly. The solutions of these nonlinear systems are dominated by their singularities: physically, a real singularity controls the local behaviour of a solution. There is a long tradition in applied mathematics to solve nonlinear problems by expansion in powers of some "small" perturbation parameter. The advantage of this approach is that it reduces the original nonlinear problem to a sequence of linear problems (Makinde, 1999). However, it is not always possible to find an unlimited number of terms of power series. Often it is possible to obtain a finite number of terms of that series and these may contain a remarkable amount of information. One can reveal the solution behavior near the critical points by analyzing partial sum (Makinde, 2001). Over the last quarter century, highly specialized techniques have been developed to improve the series summation and also used to extract the required information of the singularities

We investigate the flow of a viscous incompressible fluid in a collapsible tube. A special type of Hermit-Padé approximants technique is presented and employed to analyze the flow structure. The chief merit of this new method is its ability to reveal the dominant singularity in the flow field together with solution branches of the underlying problem in addition to the one represented by the original series.

\section{The Formulation of the Problem and Analysis}

Consider the transient flow of a viscous incompressible fluid in a collapsible tube. We take a cylindrical polar coordinate system, where oz lies along the center of the tube $(r, \theta, z), \mathrm{r}$ is the distance measured radially and $\theta$ is the azimuthal angle. Let $\mathrm{u}$ and $\mathrm{v}$ be the velocity components in the directions of $\mathrm{z}$ and $\mathrm{r}$ increasing respectively.

Assume, $\quad r=a_{0} \sqrt{1-\alpha t}$ where $\alpha$ is a constant of dimension $\left[T^{-1}\right]$ which characterizes unsteadiness in the flow field, $a_{0}$ is the characteristic radius of the tube at time $\mathrm{t}$ $=0$ as shown in the figure below.

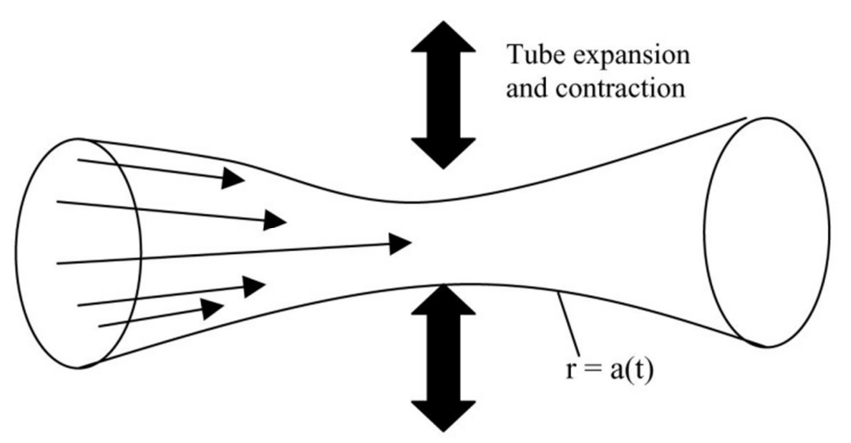

Figure 1. Collapsible tube

For axisymmetric unsteady viscous incompressible flow, the governing equations are as follows:

Continuity equation

$$
\frac{\partial(r v)}{\partial r}+r \frac{\partial u}{\partial z}=0
$$

Navier-Stokes equations

$$
\begin{gathered}
\frac{\partial u}{\partial t}+u \frac{\partial u}{\partial z}+v \frac{\partial u}{\partial r}=-\frac{1}{\rho} \frac{\partial p}{\partial z}+v \nabla^{2} u \\
\frac{\partial v}{\partial t}+u \frac{\partial v}{\partial z}+v \frac{\partial v}{\partial r}=-\frac{1}{\rho} \frac{\partial p}{\partial r}+v\left(\nabla^{2} u-\frac{v}{r^{2}}\right)
\end{gathered}
$$

Energy equation

$$
\rho c_{p}\left\{\frac{\partial T}{\partial t}+u \frac{\partial T}{\partial z}+v \frac{\partial T}{\partial r}\right\}=K \nabla^{2} T
$$


where,

$$
\nabla^{2}=\frac{\partial^{2}}{\partial r^{2}}+\frac{1}{r} \frac{\partial}{\partial r}+\frac{\partial^{2}}{\partial z^{2}}
$$

$P$ is the pressure,

$\rho$ is the density,

$\mathrm{v}$ is the kinematic viscosity of the fluid,

$T$ is the temperature,

$K$ the coefficient of thermal conductivity,

$u$ is the coefficient of viscosity and

$C_{P}$ is the specific heat capacity at constant pressure.

The appropriate boundary conditions are:

Regularity of solution along z- axis i.e.

$$
\frac{\partial u}{\partial r}=0, v=0, \frac{\partial T}{\partial r}=0, \text { on } r=0
$$

The axial and normal velocities at the wall are prescribed as:

$$
u=0, v=\frac{\partial a}{\partial t}, T=\frac{T_{0} z}{a_{0} \sqrt{1-\alpha t}}, \text { on } r=a(t)
$$

Introducing the stream function $\psi$ and vorticity $\omega$ as follows:

$$
\begin{gathered}
u=\frac{1}{r} \frac{\partial \psi}{\partial r}, \text { and } v=\frac{-1}{r} \frac{\partial \psi}{\partial z} \\
\omega=\frac{\partial v}{\partial z}-\frac{\partial u}{\partial r}=\frac{-1}{r} \frac{\partial^{2} \psi}{\partial z^{2}}+\frac{1}{r^{2}} \frac{\partial \psi}{\partial r}
\end{gathered}
$$

Eliminating pressure $p$ from (2) and (3) by using (7) and (8), we obtain

$$
\frac{\partial \omega}{\partial t}+\frac{1}{r} \frac{\partial(\psi, \omega)}{\partial(r, z)}+\frac{\omega}{r^{2}} \frac{\partial \psi}{\partial z}=v\left[\nabla^{2} \omega-\frac{\omega}{r^{2}}\right], \omega=-\nabla^{2} \psi
$$

Also, using (7) in (4), we have

$$
\frac{\partial T}{\partial t}+\frac{1}{r} \frac{\partial(\psi, T)}{\partial(r, z)}=\frac{K}{\rho c_{p}} \nabla^{2} T
$$

The boundary conditions become

$$
\begin{gathered}
\frac{\partial \psi}{\partial r}=0, \frac{\partial \psi}{\partial z}=-a \frac{d a}{d t}, T=\frac{T_{0} z}{\sqrt{1-\alpha t}} \text { and } r=a(t) \\
\frac{\partial}{\partial r}\left(\frac{1}{r} \frac{\partial \psi}{\partial r}\right)=0, \frac{\partial \psi}{\partial z}=0, \frac{\partial T}{\partial r}=0
\end{gathered}
$$

Introducing the following transformations:

$$
\eta=\frac{r}{a_{0} \sqrt{1-\alpha t}}, \psi=\frac{a_{0}^{2} \alpha z F(\eta)}{2}, \omega=\frac{-\alpha z G(\eta)}{2 a_{0}(\sqrt{1-\alpha t})^{3}}, T=\frac{T_{0} z \theta(\eta)}{a_{0} \sqrt{1-\alpha t}}
$$

Substituting (13) into equations (9)-(12), we have

$$
\begin{gathered}
\frac{d}{d \eta}\left[\frac{1}{\eta} \frac{d(\eta G)}{d \eta}\right]=R\left[\frac{G}{\eta} \frac{d F}{d \eta}-F \frac{d}{d \eta}\left(\frac{G}{\eta}\right)+\eta \frac{d G}{d \eta}+3 G\right] \\
G=\frac{d}{d \eta}\left[\frac{1}{\eta} \frac{d F}{d \eta}\right] \\
\frac{d}{d \eta}\left(\eta \frac{d \theta}{d \eta}\right)=P R\left[\theta \frac{d F}{d \eta}-F \frac{d \theta}{d \eta}+\eta^{2} \frac{d \theta}{d \eta}+\eta \theta\right] \\
\frac{d}{d \eta}\left[\frac{1}{\eta} \frac{d(\eta G)}{d \eta}\right]=0, F=0, \frac{d \theta}{d \eta}=0, \eta=0 \\
\frac{d F}{d \eta}=0, F=1, \theta=1 \text { and } \eta=1
\end{gathered}
$$

where, $R=\frac{a_{0}{ }^{2} \alpha}{2 v}$ is the Reynolds number and $P R=\frac{\rho c_{p} a_{0}{ }^{2} \alpha}{2 K}$ is a product of Prandtl number and Reynolds number (i-e Peclet number).

\section{Solution of the Problem}

By assuming a power series expansion, we solve equations (14 - 17) for small Reynolds number of the form:

$$
F=\sum_{i=0}^{\infty} F_{i} R^{i}, G=\sum_{i=0}^{\infty} G_{i} R^{i} \text { and } \theta=\sum_{i=0}^{\infty} \theta_{i} R^{i}
$$

Substituting equation (18) into equations $(14-17)$ and collecting the coefficients of like powers of $R$, we have the following Zero order and First order equations are:

Zero $^{\text {th }}$ order

$$
\frac{d}{d \eta}\left(\frac{1}{\eta} \frac{d\left(\eta G_{0}\right)}{d \eta}\right)=0, G_{0}=\frac{d}{d \eta}\left(\frac{1}{\eta} \frac{d\left(F_{0}\right)}{d \eta}\right)=0, \frac{d}{d \eta}\left(\eta \frac{d\left(\theta_{0}\right)}{d \eta}\right)=0
$$

With boundary conditions

$$
\frac{d}{d \eta}\left(\frac{1}{\eta} \frac{d\left(F_{0}\right)}{d \eta}\right)=0, \mathrm{~F}_{0}=0, \frac{d \theta_{0}}{d \eta}=0 \text { on } \eta=0
$$

$$
\frac{d F_{0}}{d \eta}=0, \mathrm{~F}_{0}=1, \theta_{0}=1 \text { on } \eta=1
$$

First order 


$$
\begin{gathered}
\frac{d}{d \eta}\left(\frac{1}{\eta} \frac{d\left(\eta G_{1}\right)}{d \eta}\right)=\frac{G_{0}}{\eta} \frac{d F_{0}}{d \eta}-F_{0} \frac{d}{d \eta}\left(\frac{G_{0}}{\eta}\right)+\eta \frac{d G_{0}}{d \eta}+3 G_{0} \\
\mathrm{G}_{1}=\frac{d}{d \eta}\left(\frac{1}{\eta} \frac{d F_{1}}{d \eta}\right) \\
\frac{d}{d \eta}\left(\eta \frac{d \theta_{1}}{d \eta}\right)=P\left[\theta_{0} \frac{d F_{0}}{d \eta}-F_{0} \frac{d \theta_{0}}{d \eta}+\eta^{2} \frac{d \theta_{0}}{d \eta}+\eta \theta_{0}\right]
\end{gathered}
$$

With boundary conditions

$$
\frac{d}{d \eta}\left(\frac{1}{\eta} \frac{d F_{1}}{d \eta}\right)=0, \mathrm{~F}_{1}=0, \frac{d \theta_{1}}{d \eta}=0, \text { on } \eta=0
$$

Solution of Zero ${ }^{\text {th }}$ order

$$
\begin{aligned}
\mathrm{F}_{0}=2 \eta^{2}- & \eta^{4} \\
F(\eta)= & \left(2 \eta^{2}-\eta^{4}\right)+\frac{R \eta^{2}}{36}\left(\eta^{2}-1\right)^{2}\left(\eta^{2}-10\right)-\frac{R^{2} \eta^{2}}{10800}\left(\eta^{2}-1\right)^{2}\left(2 \eta^{6}-101 \eta^{4}+\right. \\
& \left.+596 \eta^{2}-1057\right)+\frac{R^{3} \eta^{2}}{19051200}\left(\eta^{2}-1\right)^{2}\left(39 \eta^{10}-1287 \eta^{8}+33108 \eta^{6}-\right. \\
& \left.-195627 \eta^{4}+539468 \eta^{2}-731546\right)+O\left(R^{4}\right) . \\
G(\eta)= & -8 \eta+\frac{2 R \eta}{3}\left(2 \eta^{4}-12 \eta^{2}+7\right)-\frac{R^{2} \eta}{135}\left(3 \eta^{8}-105 \eta^{6}+408 \eta^{4}-705 \eta^{2}+\right. \\
& +271)+\frac{R^{3} \eta}{113400}\left(52 \eta^{12}-1365 \eta^{10}+25515 \eta^{8}-125300 \eta^{6}+275380 \eta^{4}-\right. \\
& \left.-286587 \eta^{2}+95360\right)+O\left(R^{4}\right) . \\
\theta(\eta)= & 1-\frac{P R}{4}\left(\eta^{2}-1\right)\left(\eta^{2}-4\right)+\frac{P R^{2}}{288}\left(\eta^{2}-1\right)\left(\left(\eta^{2}-1\right)^{2}\left(\eta^{2}-13\right)-\right. \\
- & \left.P\left(32 \eta^{4}-175 \eta^{2}+257\right)\right)-\frac{P R^{3}}{259200}\left(\eta^{2}-1\right)\left[4 ( \eta ^ { 2 } - 1 ) ^ { 2 } \left(\eta^{6}-60 \eta^{4}+\right.\right. \\
+ & \left.417 \eta^{2}-918\right)+P\left(25 \eta^{10}-794 \eta^{8}+9781 \eta^{6}-27669 \eta^{4}+\right. \\
+ & \left.\left.33306 \eta^{2}-23169\right)\right)+9 P^{2}\left(64 \eta^{8}+714 \eta^{6}-8011 \eta^{4}+2271 \eta^{2}-\{\}\right. \\
- & 22261)]+O\left(R^{4}\right)
\end{aligned}
$$

\section{Conclusion}

We observed that the fluid axial velocity profile is parabolic with maximum value at centerline and minimum at the plates. It is interesting to Axial velocity profile during tube con-traction $(R>0)$.

Note that the fluid axial velocity generally declines with rise in tube contraction due to the strong influence of the negative transmural pressure owing to marked reduction of rigidity (i.e. $R>0$ ). In reality, this is likely, since contraction brings about a reduction in the tube's cross-sectional area, hence, decreasing the amount of flow passing through the compressed region. Table 5.01, shows the convergence of the dominant singularity $R_{c}$ in the flow field together with its corresponding exponent $\alpha_{c}$, as well as the asymptotic performance of wall skin friction and pressure gradient as the flow Reynolds number tends to zero. It is noteworthy also that $R_{c}$ is the bifurcation point and lies in the negative real axis of the flow Reynolds number $R$, i.e., the area representing tube's contraction. This critical value of Reynolds number enables the biomedical engineers to determine accurately the maximum expansion of the tube walls due to the variation in the tube's external and internal pressure i.e., $a_{0}=2 v R_{c} / \alpha$. Figures 2 and 3 show the sketch 
of bifurcation diagrams for the skin friction and fluid axial pressure gradient parameters. For tube's contraction i.e., $R>0$, only one solution branch exist i.e., type I. This is the main solution branch and it shows that the wall skin friction and fluid axial pressure gradient increase with increase in $R$. In the expansion region i.e., $R<0$, two solution branches are identified (i.e., type I, II). A simple turning point with exponent $\alpha_{c}=0.5$ exits between type I and type II solution branches i.e., $R_{c}$. We observed that the type II solution is physically unreasonable but mathematically interesting. It is interesting to note that the turning point here also corresponds to the dominant singularity in the flow field.

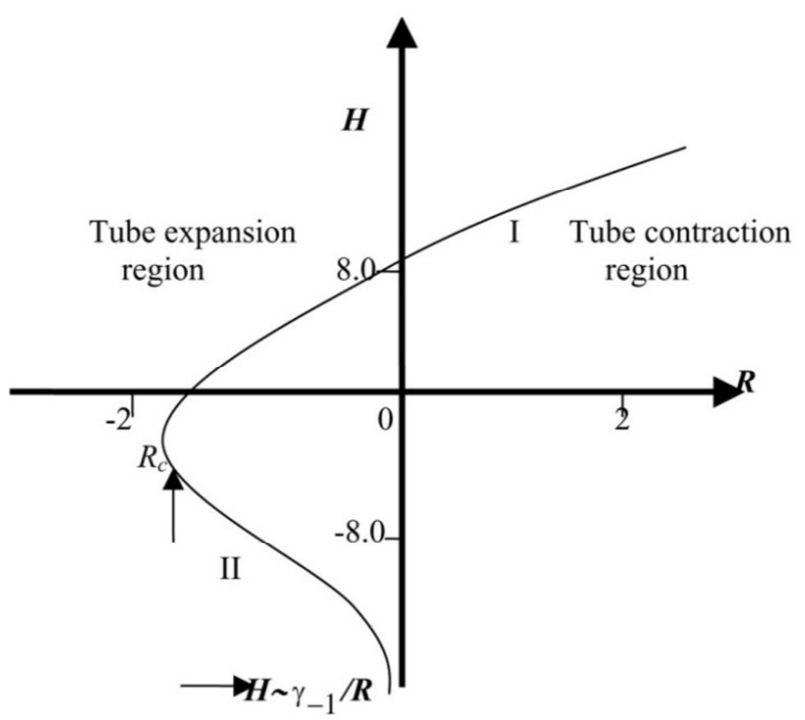

Figure 2. A sketch of bifurcation diagram for skin friction.

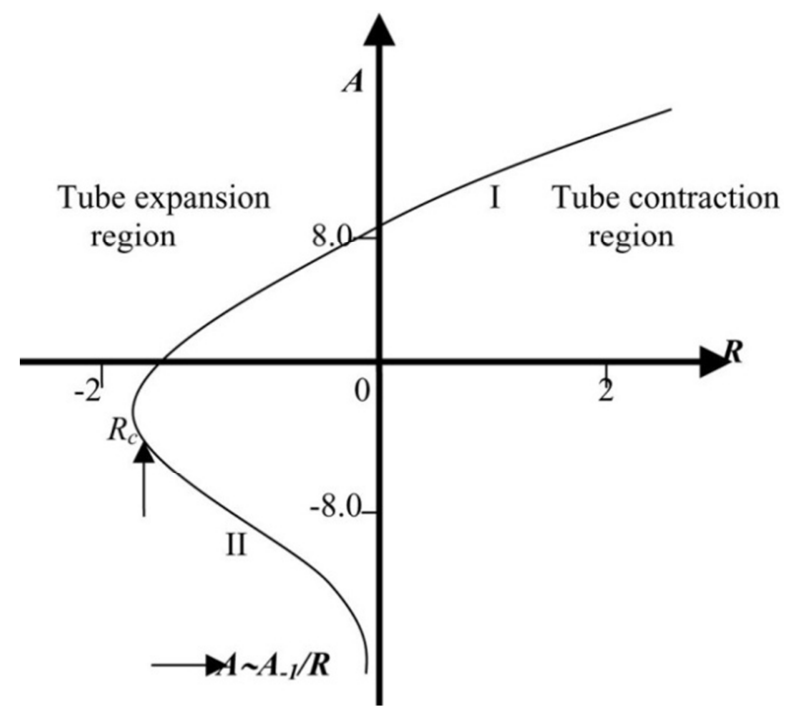

Figure 3. A sketch of bifurcation diagram for axial pressure gradient.

Finally, in this paper, we have proposed a new form of series summation and improvement technique based on the generalizations of Padé approximants i.e., a special type of Hermit-Padé approximant. We have applied this method to investigate the problem of squeezing flow in parallel plate's viscometer with great success. The chief novelty of this procedure is its ability to reveal the dominant singularities together with solution branches of the underlying nonlinear problem in addition to the branch represented locally by the original series. Generally, we have found that this new method is very competitive. However, we have not yet develop a theory that would explain its strengths and limitations and so we have relied on intelligent numerical investigation.

\section{Appendix} (26) (26).

A1: The Maple procedure to solve the equations (23) to

\# Here we declare the arrays to store the computed results $\mathrm{F}:=\operatorname{array}(0 . .34): \mathrm{G}:=$ array $(0 . .34):$ Fr:=array $(0 . .34)$ : Gr:=array $(0 . .34)$ :

\# Here we input the zero order solution $F[0]$ and $G$ [0]. F $[0]:=\left(2 * r^{\wedge} 2-r^{\wedge} 4\right): G[0]:=-8 * r$ :

$\operatorname{Fr}[0]:=\operatorname{diff}(\mathrm{F}[0], \mathrm{r}): \operatorname{Gr}[0]:=\operatorname{diff}(\mathrm{G}[0], \mathrm{r})$ :

\# This computes the higher order teams i.e. $\mathrm{n}>0$. for $\mathrm{n}$ from 1 by 1 to 43 do

A1:=normal $\left(1 / \mathrm{r}^{*} \operatorname{sum}(\mathrm{g}[\mathrm{i}] * \mathrm{fr}[\mathrm{n}-\mathrm{i}-1]+\mathrm{f}[\mathrm{i}] *(\mathrm{~g}[\mathrm{n}-\mathrm{i}-1] / \mathrm{r}-\right.$ gr $[n-i-1]), i=0 . . n 1)): A 2:=$ normal $\left(r^{*}\right.$ gr $\left.[n-1]+3 * g[n-1]\right)$ : $\mathrm{A}:=\mathrm{R} *(\mathrm{~A} 1+\mathrm{A} 2)$ :

A1: $=0:$ A2: $=0:$ g11: $=$ normal $\left(r^{*}(\right.$ int $\left.(A, r)+K)\right): A:=0$ : $\mathrm{g} 1:=$ normal $($ int $(\mathrm{g} 11, \mathrm{r}) / \mathrm{r})$ :

$\mathrm{g} 11:=0: \mathrm{fl1}:=\operatorname{normal}\left(\mathrm{r}^{*}(\right.$ int $\left.(\mathrm{g} 1, \mathrm{r})+\mathrm{M})\right): \mathrm{fl}:=$ normal (int $(\mathrm{f} 11, \mathrm{r}))$ :

$\mathrm{r}:=1: \mathrm{K}:=$ normal $($ solve $(\mathrm{f} 11=0, \mathrm{~K}): \mathrm{M}:=$ normal $($ solve $(\mathrm{fl}=0, \mathrm{M})): \mathrm{r}:={ }^{\prime} \mathrm{r}$ :

$\mathrm{fl1}:=0: \mathrm{F}[\mathrm{n}]:=\operatorname{normal}(\mathrm{fl}): \mathrm{fl}:=0: \mathrm{G}[\mathrm{n}]:=\operatorname{normal}(\mathrm{g} 1)$ :

$\mathrm{g} 1:=0: \operatorname{Fr}[\mathrm{n}]:=$ normal $(\operatorname{diff}(\mathrm{F}[\mathrm{n}], \mathrm{r})):$ Gr $[\mathrm{n}]:=$ normal

$(\operatorname{diff}(\mathrm{G}[\mathrm{n}], \mathrm{r}))$ : $\mathrm{K}:={ }^{\prime} \mathrm{K}$ : $: M:={ }^{\prime} \mathrm{M}^{\prime}$ : print $(\mathrm{F}[\mathrm{n}])$; print $(\mathrm{G}$ $[\mathrm{n}])$;

\# Here we compute the wall skin friction coefficients. print (evalf (sub (r=1, G [n])));

od: quit ();

\section{References}

[1] C. D. Bertram, (1986), Unstable equilibrium behaviour in collapsible tubes, J. Biomech. 19, 61-69.

[2] C. D. Bertram and T. J. Pedley, (1982), A mathematical model of unsteady collapsible tube behaviour, J. Biomech. 15, 39-50.

[3] C. D. Bertram and C. J. Raymond, (1991), Measurements of wave speed and compliance in a collapsible tube during selfexcited oscillations: A test of the choking hypothesis, Med. Biol. Eng. Comput. 29, 493-500.

[4] C. D. Bertram, C. J. Raymond and T. J. Pedley, (1990), Mapping of instabilities for flow through collapsible tubes of deferring length, J. Fluids. Struct. 4, 125-153.

[5] C. D. Bertram, C. J. Raymond and T. J. Pedley, (1991), Application of nonlinear dynamics concepts to the analysis of self-excited oscillations of a collapsible tube conveying a fluid, J. Fluids. Struck. 5, 391-287. 
[6] M. Bonis and C. Ribreau, (1987), Etude de quelques propriétés de l'ecoulement dans une conduite collabable, $L a$ Houille Blanche 3/4, 165-173.

[7] R. W. Brower and C. Scholten, (1975), Experimental evidence on the mechanism for the instability of flow in collapsible vessels, Med. Biol. Engng 13, 839-845.

[8] W. A. Contrad, (1969), Pressure-flow relationship in collapsible tubes, IEEE Trans. Bio-Med. Engng BME-16, 284 295.

[9] S. J. Cowley, (1982), Elastic jumps in fluid-filled elastic tubes, J. Fluid Mech. 116, 459-473.

[10] S. J. Cowley, (1983), On the wavetrains associated with elastic jumps on fluid-filled elastic tubes, $Q$. J. Mech. Appl. Math., $36,289-312$.

[11] C. Domb and M. F. Sykes, (1957), On the susceptibility of a ferromagnetic above the Curie point, Proc. R. Soc. London, Ser. A, 240, 214-228.

[12] P. G. Drazin and Y. Tourigny, (1996), Numerical study of bifurcations by analytic continuation of a function defined by a power series, SIAM J. Appl. Math. 56, 1-18.

[13] D. Elad, R. D. Kamm and A. H. Shapiro, (1987), Choking phenomena in a lung-like model, ASME J. Biomech. Engng 109, 1-9.

[14] J. E. Flaherty, J. B. Keller and S. I. Rubinow, (1972), Post buckling behaviour of elastic tubes and rings with opposite sides in contact, SIAM J. Appl. Math., 23, 446-455.
[15] J. B. Grotberg, (1971), Pulmonary flow and transport phenomena, Ann. Rev. Fluid Mech. 26, 529-571.

[16] A. J. Guttamann, (1989), Asymptotic analysis of power series expansions, Phase Transitions and Critical Phenomena, C. Domb and J. K. Lebowitz, eds. Academic Press, New York, $1-234$.

[17] C. Hunter, C. and B. Guerrieri, (1980), Deducing the properties of singularities of functions from their Taylor series coefficients, SIAM J. Appl. Math., 39, 248-263.

[18] D. L. Hunter and G. A. Baker, (1979), Methods of series analysis III: Integral approximant methods, Phys. Rev. B 19, 3808-3821.

[19] M. Heil, (1997), Stokes flow in collapsible tubescomputational and experiment, J. Fluid Mech., 353, 285-312.

[20] O. D. Makinde, (1999), Extending the utility of perturbation series in problems of laminar flow in porous pipe and a diverging channel, Jour. of Austral. Math. Soc. Ser. B 41, 118 128 .

[21] O. D. Makinde, (2001), Heat and mass transfer in a pipe with moving surface: Effects of viscosity variation and energy dissipation, Quaestiones Mathematicae, Vol. 24, 97-108.

[22] R. E. Shafer, (1974), On quadratic approximation, SIAM J. Numer. Anal., 11, 447-460. 